\title{
Perspectives on Wound Healing
}

\author{
Weledji EP* \\ Department of Surgery, Faculty of Health Sciences, \\ University of Buea, Cameroon \\ *Corresponding author: Weledji EP, Department of \\ Surgery, Faculty of Health Sciences, University of Buea, \\ Cameroon
}

Received: August 01, 2017; Accepted: August 23, 2017; Published: August 31, 2017

\begin{abstract}
Wound healing is an important component of all surgical operations. Essential features of the healing process are common to wounds of all tissues. Healing requires restoration of epithelial continuity with restoration of strength in the supporting dermis. In addition to the complications from delayed wound healing, there is a negative psychological effect on the patient. In the adult mammal, injury to the stroma is typically irreversible and eventually leads to formation of a scar, a non-physiological tissue repair. Regeneration of stroma and recovery of the structure and function of the original tissue is typically not observed. This article reviewed the fundamental process of wound healing, the factors that delay wound healing and their clinical implications.
\end{abstract}

Keywords: Wound; Healing; Delayed; Clinical implications

\section{Introduction}

A wound is a disruption of the normal continuity or contiguity of body structures caused by physical injury. The wounding agent may penetrate the surface epithelium, or give rise to a non-penetrating wound in which the integument remains intact while the force is transmitted to subcutaneous tissues or viscera. A classification according to the mode of damage will include an incised wound caused by a sharp instrument and when there is associated tissue tearing it is lacerated. An abrasion results from friction damage to the body surface and causes superficial bruising and loss of epithelium. A crush injury may be associated with massive tissue destruction without breaching the skin. Gunshot wounds are caused by pellets or bullets, and bullets fired from high-velocity rifles cause much more destruction than low-velocity bullets. Burns are a distinct variety of wounds due to heat, cold, electricity, irradiation or chemicals. The biological objectives of wound healing are to restore the integrity of the epithelial surfaces should this have been lost and to restore the tensile strength of the sub-epithelial tissue (dermis) [1]. Wound healing may be summarized into 3 phases. An initial lag phase which is the inflammatory response to injury where capillary permeability increases and protein-rich exudates (fibrin) forms in wound, while inflammatory cells migrate into the area. There is a delay of 2-3 days before fibroblasts begin to manufacture collagen from the proteinrich exudates (lag phase). This is followed with the incremental phase in which cell proliferation, progressive collagen synthesis (matrix formation) and gain in tensile strength occurs. The third phase is the plateau phase of matrix remodeling [2-4].

\section{Epidermal and Dermal Events}

From the point of incision, haemorrhage lets platelets; fibrinogen and fibronectin accumulate in the wound acting as a tissue glue. Epithelial cells migrate over the raw area and proliferate. The dermal events include collagen formation and wound contraction. In the dermis an acute inflammatory response causes an influx of neutrophils and macrophages in the first two days which removes tissue debris. Fibroblasts proliferate and secrete collagen to restore tensile strength. Macrophages lying adjacent to fibroblasts may provide the amino acids (proline and lysine) essential for collagen formation in the endoplasmic reticulum of fibroblasts. Oxygen and ascorbic acid (vitamin c) are necessary for their hydroxylation and incorporation into tropocollagen which leaves the fibroblasts and polymerizes in between the cells to form collagen. Fibroblasts synthesize mucopolysaccharide ground substance for alignment and approximation of the tropocollagen monomers prior to polymerization [2-4]. They also secrete and attach to fibronectin via $\alpha 5 \beta 1$ integrin within 3-D fibrin matrices, resulting in the formation of localized fibronectin tracks. Other cells use these fibronectin tracks as conduits, resulting in an interconnected cell-fibronectin network. Interfering with cell-fibronectin binding with RGD peptide, anti $\alpha 5$ integrin or anti fibronectin antibodies inhibited cell spreading and migration through fibrin, but did not affect cell behavior in collagen [5]. Topically applied fibronectin was found to significantly improve wound healing in irradiated skin and was associated with decreased inflammatory infiltrate and increased angiogenesis [6]. Collagen synthesis demands a continuous supply of energy. The ingrowths of capillary beds with the fragile capillary arteries bring oxygen and nutrients to the wound. A local stimulus for collagen synthesis in fibroblasts is high lactate levels consequent on ischemia [7]. In unopposed wounds granulation tissue (mixture of capillaries, fibroblasts, macrophages and leucocytes) which is red, granular, friable and bleeds to touch is a major feature. Myofibroblasts contain micro fibrils which enable them to pull in the wound margins. This wound contraction takes place from days $2-3$ and reduces the energy needed for wound healing by reducing the size of the defect in unopposed wounds. However, the excessive shrinkage of fibrosis may cause unsightly puckering and restricted mobility of the wound crossing a joint (contracture). Excessive collagen is removed leaving an interlocking network of collagen rather than an amorphous mass. This is associated with decrease in fibroblasts and inflammatory cells (matrix remodeling). The wound continues to gain in tensile strength for some 6 months, although the gain over the first 7- 10 days is usually sufficient to allow removal of skin sutures without wound disruption. However, remodeling never returns the wound to normal, and skin and fasciae cover only $80 \%$ of their original tissue strength [2-4].

\section{Growth Factors and Cytokines}

Growth factors (GFs) are peptides that reach their target through 

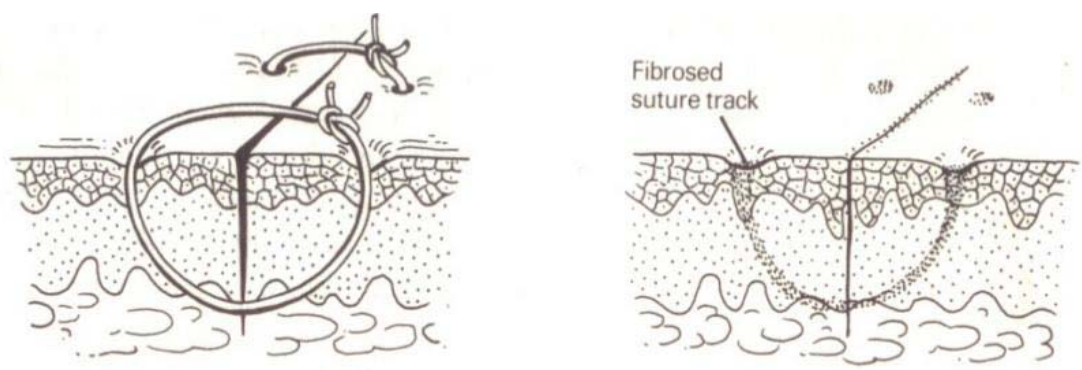

a) Primary Intention

Figure 1: Healing by first intention [3]
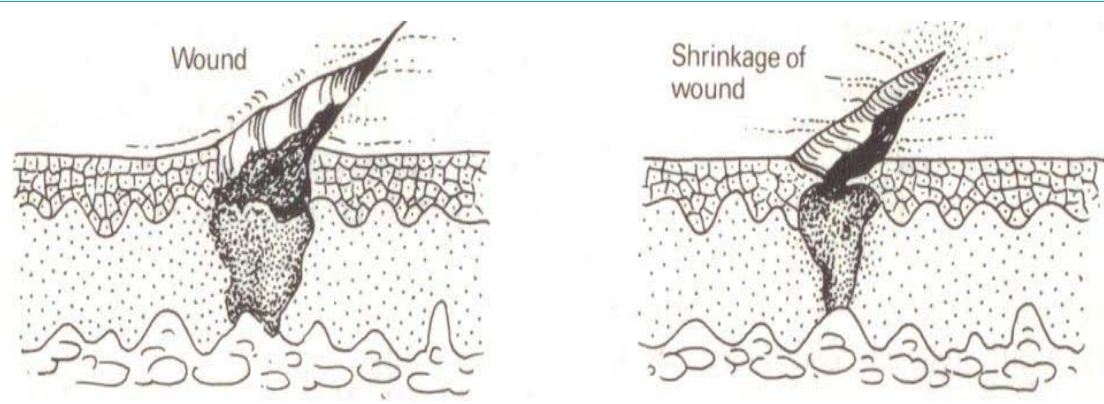

Figure 2: Healing by second intention [3].

the endocrine, paracrine or autocrine pathways. They work on stem cells stimulating mitogenesis. Important examples include Platelet Derived Growth Factors (PDGF) synthesized by platelets, macrophages and endothelial cells. By attracting inflammatory cells (neutrophils, macrophages), fibroblasts, initiating proliferation of fibroblasts, smooth muscle and endothelial cells, increasing collagen synthesis and modulating synthesis of other GFs, they are important in the regulation of all stages of wound repair. Epidermal Growth Factor (EGF) and transforming growth factors $\alpha$ and $\beta$ are important mediators of wound healing. Cytokines are a group of low molecular weight protein cell regulators that include lymphokines, monokines, interleukins and interferons. They are produced rapidly and locally usually acting in a paracrine or autocrine manner. IL- 1 and $\alpha$ TNF (cachectin) are important cytokines involved in wound healing as they have proliferative effects on dermal fibroblasts, stimulating collagen synthesis and the ingrowths of new blood vessels in healing wounds [4-9]. The disadvantages of using growth factors are: cost of treatment, treatment may not be available and the application method. It may need to be injected into the wound, and the choice of which growth factor, when, and what dosage is a problem [10]. Growth factors are obviously not an alternative to good wound care.

\section{The Balance of Forces in Wound Healing}

There are competing influences in wound healing. The laying down of fibrous tissue and the digestion of this tissue to modify the shape of the scar are normally in balance. If little fibrous tissue is laid down or it is digested excessively, then dehiscence is likely. This can be predicted in patients who have undergone radiotherapy at the wound site, in patients on steroid medication and in patients with Ehlers-Danlos syndrome, or diabetes. In other circumstances, deposition of fibrous tissues is excessive and results in a heaped- up, hypertrophic or keloid scar [11,12]. Although only humans are affected, the risk factors for a hypertrophic/ keloid scar are genetic (especially Negroes), tension on the wound, delayed healing and the site of the scar [12-14].

The balance of forces in wound healing

Collagen synthesis

Wound Dehiscence

Collagen breakdown

Wound closure may also be viewed as occurring by a small number of processes, namely contraction, scar formation, and regeneration rather than its intermediate mechanism involving biochemical and cell-biological interactions. Scar formation has been frequently cited as a physical and chemical barrier for regeneration particularly in studies of regeneration of axons in the injured central nervous system. However, in studies with wounds in skin and peripheral nerves scar formation can be cancelled and regeneration achieved by appropriate modification of the wound healing process, particularly blocking of the normal wound contraction process [15].

\section{Healing in Some Specialized Tissues Skin}

Healing by first (primary) intention is the ideal method of healing. It requires minimal expenditure of energy and results in a fine hairline scar (Figure 1). The meticulous apposition of the edges of a clean incised wound leaves a narrow epidermal defect which can be bridged easily. The basal epidermal cells at the wound edges undergo mitosis and continuity of the basal layer is restored within a few days.

Healing by second intention occurs if the wound edges are 
not opposed. The defect fills gradually with granulation tissue and restoration of epidermal continuity may take considerable time (Figure 2). Continued basal cell division at the wound edges produces sheets of cells which migrate across the denuded area but their advance is often hindered by infection. Thus, healing by second intention usually results in prolonged healing, excessive fibrosis and an ugly puckered scar, or a hypertrophic/ keloid scar [1-4]. Scar formation depends critically on wound contraction and is, therefore, a healing process secondary to contraction [15]. It may be possible to compromise healing by second intention by refreshening the wound edges and bringing them into apposition or by covering the defect with a skin graft.

\section{Bone}

The essential difference between healing of a fracture and softtissue injury is preliminary cartilage formation and subsequent conversion of fibro cartilage to bone. The fibroblast still retains central importance as its collagen provides basic framework on which hydroxyapatite crystals condense to form bone. Torn blood vessels produce a haematoma between the bone ends and fibroblasts and capillaries grow into the ischaemic area to form granulation tissue. Leucocytes and macrophages are soon at hand (day 3-7) to destroy bacteria and phagocytes debris. Cartilage forms within the granulation tissue and the resulting elastic connective tissue or callus binds the bone ends together. Dormant osteogenic cells in the periosteum become active osteoblasts and lay down a sheath of bone around the broken ends, and at the same time new bone forms in the medullary cavity. The cartilage in the callus calcifies and is replaced by a process of intra membranous bone formation. The callus gradually shrinks and the new bone between the fracture ends becomes compact rather than spongy, with ultimate bony union. Extensive remodeling follows and it becomes difficult to identify the fracture site. Strength returns gradually over the months [16].

\section{Nervous tissue}

Wounds of the nervous system involve both neural and connective tissue. Neural tissue in the Central Nervous System (CNS) cannot regenerate as a result of the inhibitory action of the CNS glial cells. Fibroblasts derived from glial or perivascular cells replace nervous tissue with collagen. Damage to the peripheral nervous system is not so final. Cut axons degenerate (Wallerian) distal to the injury but provided the neural body survives the axons of the proximal stump attempt re-innervations by sprouting and crossing the gap to enter the empty Schwann tube. This is aided by fibroblasts forming a connecting bridge although this may prove self-defeating if it results in a dense collagen scar. Not all the axons find their way into Schwann tubes. Some enter into a wrong tube and some perish as a result of entering tubes already occupied. Nevertheless, useful re-innervations is possible as regenerating axons can grow as quickly as $4-5 \mathrm{~mm} /$ day $[17,18]$.

\section{Gastrointestinal tissue}

Damage confined to gut mucosa, as in gastric erosion, is repaired by re-epithelisation and leaves no scar. A return to normal becomes impossible when ulceration extends through the sub mucosa to involve underlying muscles and a permanent fibrous scar is inevitable. The stomach and small bowel have a rich blood supply and contain relatively small number of pathogenic bacteria. Because of easy

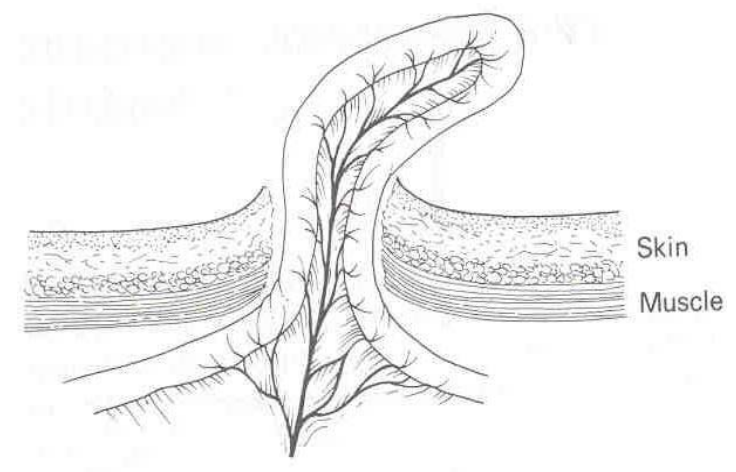

Figure 3: Schematic diagram of evisceration (burst abdomen) [25].

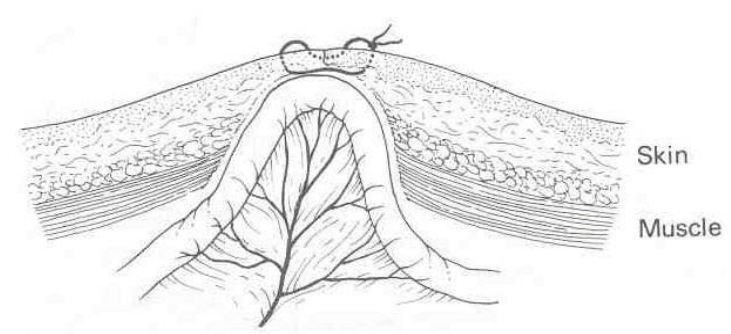

Figure 4: Schematic diagram of incisional hernia [24].

sealage unlike the biliary and urinary system, leakage is uncommon following resection and anastomosis. Lysis of collagen occurs in the normal bowel on either side of the anastomosis as part of the inflammatory response but at 1 week, the anastomosis is usually able to withstand disruption. Thus, early anastomotic disruption is usually from technical failure and manifest clinically by the $4^{\text {th }}-6^{\text {th }}$ postoperative day. Late anastomotic disruption (>1 week) is usually due to biological (healing) failure [19]. The oesophagus in addition to the lack of a serosal layer has a weaker wall than the stomach and proximal intestine and has less extensive blood supply [20].The distal colon has tenuous blood supply and here the problems of healing are increased by the large number of pathogenic bacteria in the bowel lumen [19]. Bacteria delay collagen synthesis and collagenase production can cause excessive Lysis. Surgical technique is thus of fundamental importance in reducing the incidence of anastomotic leakage and wound infection after gastro-intestinal surgery [21,22]. The connective tissue of the sub mucosa is the strongest layer of the bowel wall and sutures or staples in this layer maintain apposition until sufficient tensile strength has been regained [21,23].

\section{Wound Strength and Wound Faillure}

The strength of a wound is due initially to the suture which is greatest on the day of operation and progressively declines. To this is added, wound strength from healing which is zero on day of operation and progressively increases after a lag phase [24]. Thus, wound failure can be due to a mechanical failure or a healing (biological) failure.

\section{Wound failure}

A mechanical failure is because the wound is not held together long enough. This may be due to suture failure (inadequate materials e.g. absorbable instead of non-absorbable, inadequate strength, knot 
failure or inappropriate technique e.g. too small bites leaving a suture too short to stretch when the wound stretches (should obey Jenkins's rule of 4 tol suture to abdominal wound length); mechanical disruption or excessive mobility: coughing, ascites, obstruction leading to distension [25]. Excess mobility may occur e.g. on a poorly immobilized fractured long bone.

A healing (biological) failure may result from factors local to the wound (haematoma, tissue damage from trauma or diathermy, necrotic tissue, local infection, foreign material (e.g. excessive diathermy, sutures), tumour cells in wound, poor blood supply (because of site, tension or previous radiotherapy), irradiated tissues, obesity and continuing trauma to wound. Factors general to the body (systemic) which would affect any wound that was made would include old age, malnutrition, vitamin deficiency especially vitamin $\mathrm{C}$, trace metal deficiency especially zinc, anemia, uremia jaundice, diabetes mellitus, generalized infection, shock leading to poor tissue perfusion, cytotoxic drugs, steroids and AIDS.) [25,26] From all available evidence, old age, malnutrition, vitamin deficiency, anaemia and malignancy are doubtful factors that delay wound healing. The elderly are a heterogenous group and it is important to distinguish chronological from physiological age. Malnutrition has to be severe before healing is affected because the wound has high priority when competing with unwounded tissue for body resources [26]. Anaemia per se does not impair healing if normovolaemic and normal $\mathrm{O}_{2}$ tension. The factors that probably delay wound healing are severe malnutrition, uremia and cytotoxic drugs whereas, the factors that definitely delay wound healing are zinc deficiency, trauma, hypovolaemia (hypo albuminaemia), hypoxia (arterial and venous insufficiency), jaundice, steroids, diabetes mellitus and AIDS [27,28].

Many of these factors are inter-related, such as the site of the wound, the blood supply and level of tissue oxygenation. Wounds of the head and neck heal quickly and sutures or skin clips can be removed within 3-5 days. Wounds of the leg and feet heal slowly and sutures are retained for 14 days. The differences are due to blood supply as warm, vascular areas are generally quicker to heal than cool extremities. In addition, hypoxia compromises the ability of leucocytes to kill bacteria and also favours the growth of potentially dangerous anaerobic organisms. Wounds heal less well with advancing age due to a combination of diminished tissue response to injury, impaired blood supply from arteriosclerosis, poor nutritional status and the high incidence of inter current disease e.g. diabetes mellitus, COAD) $[23,28]$.

Specific factors: The two main specific factors that affect wound healing are blood supply and infection. Wounds heal slowly in ischaemic tissue. They are prone to infection and failure to heal by first intention. In addition the ischaemic wound may not be able to sustain the metabolic demand necessary for healing by second intention. The arterial $\mathrm{O}_{2}$ partial pressure $\left(\mathrm{PO}_{2}\right)$ is the important determinant of therate of collagen synthesis. The local tissue hypoxia from chronic venous hypertension/insufficiency predisposes the venous ulcer at the gaiter area which can be relieved by stripping of the incompetent saphenous vein. The healing of ischaemic ulcers is improved by arterial revascularization. Factors which determine whether a surgical wound becomes infected are; a) general patient factors such as increasing age, cardiovascular and respiratory status, nutritional Status and the presence of intercurrent infection; b) Local wound factors entailing skin preparation and meticulous aseptic technique (sterilization and antisepsis) minimize bacterial contamination of the wound $[28,29]$.

\section{Delayed wound healing}

Delayed wound healing is a delay in restoring the epithelial integrity of a wound or the tensile strength of the sub epithelial tissue. Delayed wound healing usually refers to an incised wound such as an abdominal incision that disrupts with the epithelial layer opened within a few days (dehiscence) or after weeks or months with partial breakdown of the fascia and muscle layers but with epithelial layer not opened (incisional hernia).

The 'Burst' Abdomen: With an incidence of about 6\%, it occurs when the whole wound breaks down with the epithelial layer open resulting in evisceration (protrusion of loops of bowel) (Figure 3). It manifests within a few (4-10) days after operation with preliminary signs of a serosanguinous discharge, sudden increase in pain, shock and distress. The cause may be classified into (a) preoperative due to the premorbid state of the patient. Poor tissues from uremia, jaundice and anaemia tear and this is exacerbated by raised abdominal pressure from obesity, coughing, constipation, ascites and urinary retention; (b) operative due to surgical technique i.e. mostly failure of suture technique, sutures break (pulling the sutures too tight, inserting sutures too close to the edge), knots slip (insecure knots); (c) postoperative wound infection causing wound disruption from sepsis. Mass closure of laparotomy wounds following Jenkins' rule decrease the incidence of the 'burst' abdomen [21-25].

Incisional hernia: It is the partial breakdown of the muscle layers with the epithelial layer not opened (Figure 4). There is a bulge (weakness) around the wound scar due to weakened fascia of the abdominal wall which may occur months after the operation. The incidence is about $8 \%$ and the causes are (1) infection which may even be low grade, (2) poor sutures e.g. absorbable (vicryl) used instead of non-absorbable (nylon) for abdominal wound closure especially with the lesser collagen synthesis in Caucasians than in negroes [25,26,29].

Sometimes delayed wound healing may be manifest by an intestinal anastomosis leaking or a wound that is left to heal by second intention failing to close e.g. a leg ulcer, or if malignancy develops from a chronic dysplasia (Marjolin's ulcer). Sometimes a wound may fail to close completely because of continuing discharge from a chronic sinus.

\section{Factors that can be influenced to promote wound healing}

These are the nutritional status, surgical technique and intercurrent disease.

Nutritional status: Protein availability is the most important factor as wound dehiscence and infection are common when the serum albumin is low. Healing problems can be anticipated when recent weight loss is greater than $20 \%$ of original weight [26,27]. Ascorbic acid (vitamin C) is essential for proline hydroxylation and collagen synthesis, although the number of fibroblasts is not reduced in scorbutic states (scurvy). Zinc is a component of enzymes involved in the healing process and its deficiency retards healing. However, supplements of ascorbic acid and zinc are effective when these factors are deficient but do not improve healing in normal subjects [26,30,31]. 
Surgical technique: Meticulous aseptic technique (sterilization of equipment, disinfection of hands \& surgical area) avoid wound infection and poor healing. Skin incisions are placed in lines of least tissue tension (Langer's lines- natural skin creases) provided this allows adequate exposure. This provides minimal destruction and thus heals promptly to leave a fine scar [13]. An ideal abdominal incision is one that provides easy and rapid access, the ability to be extended if necessary, safe closure with security from early dehiscence and avoidance of long-term wound failure or herniation, little pain and good cosmetic appearance [14]. Gentle tissue handling avoids wound devitalsation. Extensive dissection, excessive use of diathermy and failure to achieve homeostasis contribute to wound devitalisation. Wound devitalisation is also avoided if sutures are not placed too close together (preferably $1 \mathrm{~cm}$ apart) and too near the wound margin (preferably $1 \mathrm{~cm}$ away) or tied too tightly [14]. The choice of suture materials should be strong enough to support the wound until tensile strength is sufficient to prevent breakdown. Non-absorbable sutures like nylon may be essential in some situations such as for the aponeurotic layer of an abdominal wound and repairs of hernias. Foreign body predisposes to infections and therefore non-absorbable sutures must be inert, mono filamentous to avoid interstices for bacteria growth. A traumatic suture (roundbodied) is used when tissue resistance is low e.g. intestinal suture. A triangular suture (cutting) is used when sharp cutting edges are needed for tough tissues e.g. skin. Potentially infective sites e.g. gut lumen during colonic surgery is isolated from the wound by sterile drapes and spillage of contents avoided by careful use of a traumatic intestinal occlusion clamps. Particular care is taken in areas of reduced resistance to infection e.g. bone or areas where infection would have serious consequences e.g. brain and spinal cord. The ideal abdominal wound closure provides strength and barrier to infection. In addition, the closure should be efficient, performed without tension or ischemia, comfortable for the patient, and aesthetic. Correct apposition of the deeper layers allow the skin to fall together without tension so that skin apposition can be achieved by superficial sutures or strips allowing healing by first intention. Tension sutures even for the 'burst abdominal wound' are avoided with the use of the mass closure technique where non-absorbable sutures are passed through all layers of abdominal wall except skin (Jenkins rule).Dead spaces are avoided if possible as bleeding and exudates formation increase infection rate $[14,21,24,25,32]$.

Jenkins rule: Jenkins, a general surgeon, worked out the mathematics of wound suturing, with a little help from Professor Hugh Dudley at St Mary's Hospital, London in 1976 [25]. He knew that vertical wounds had been found to stretch by up to a third of their length in the early post-operative period, when the patient coughs or is distended. If a continuous suture takes tiny bites, it will end up only slightly longer than the wound, so if the wound increases by a third, the suture can't stretch and must cut out. Big bites produce a concertina and a suture that is considerably longer than the wound. The bigger the tissue bites used the greater the length of suture used and therefore there will be a smaller rise in tissue tension when the wound is stretched. Jenkins calculated that a 4-1 suture to wound length ratio $(1 \mathrm{~cm}$ apart and $1 \mathrm{~cm}$ from edge of wound) was needed to prevent an increase in tension that would cause sutures to cut out [25].
Wound contamination: The risk of exogenous bacterial contamination is highest in the first 5 days. Although risk is small, it is customary to protect the wound by a sterile occlusive dressing or occlusive spray e.g. Nobecutane [33]. Skin sutures and drains are potential portals for bacterial entry and should not be retained longer than necessary. Aseptic technique is valuable for redressing of wounds. It is important to note that although wound contamination does not necessarily lead to wound infection, closed wounds when contamination occurs during surgery remain vulnerable for 3-4 days to common infecting organisms such as staphylococci, streptococci, E-coli, anaerobes (bacteroides) and anaerobic streptococci. Antibiotics are no substitute for thorough intra abdominal and wound lavage with warm saline. Wound infection may require opening of the wound for toileting and dressing followed by secondary closure if necessary [34].

Artificial and living skin equivalents: Topical antimicrobials decrease the rate of colonization of a wound, but do not prevent microbial invasion and proliferation. Therefore it is essential to close large wounds especially burn wounds by skin grafting after early wound excision and removal of toxic nonviable tissue. Depending on its composition, tissue- engineered skin can facilitate cell proliferation, production of extracellular matrix components and increase local concentrations of growth factors in the wound. Skin substitutes include epidermal components, e.g. Vivoderm, dermal components, e.g. Dermograft, and composite grafts. i.e. those containing epidermal and dermal components, e.g. Apligraf. No synthetic dermal replacement has been found to equal allograft dermis in closing wounds $[35,36]$.

Intercurrent disease: Cachetic patients from severe malnutrition and advanced cancer have marked impairment of healing. Diabetes mellitus impairs healing by reducing tissue resistance to infection (decrease neutrophil function), peripheral vascular insufficiency, and peripheral neuropathy causing decrease peripheral sensation allowing trauma to the healing wound. Hemorrhagic diathesis increase risk of haematoma formation and wound infection. The lower PO2 in chronic obstructive airway disease and the strain placed on wound during coughing affects healing and pre-disposes abdominal wound dehiscence. Corticosteroid therapy reduces the inflammatory response to injury, impairs collagen synthesis and reduces resistance to infection. Immunosuppressive therapy impairs healing by predisposing infection in patients already compromised. Compounded with the severe malnutrition in HAART-naïve AIDS patients, a low CD 4 count of less than 500 will render them susceptible to delayed wound healing [24-32].

\section{Conclusion}

Wound healing is a highly complex chain of events, and the clinicians' armamentarium continues to expand with methods of management. Cell culture models are invaluable tools to promote our understanding of the mechanisms that regulate the wound healing process and provide a platform for drug discovery. The pathway of healing is determined by characteristics of the wound on initial presentation. The most important risk factor for delayed wound healing is prolonged inflammation from whatever cause e.g. foreign body, infection, burn, inadequate wound closure, or in areas of chronic inflammation. The appropriate method selected to treat 
the wound should be based on its ability to avoid hypoxia, tension, infection, excessive edema, and foreign bodies.

\section{References}

1. Stadelmann W, Digenis AG, Tobin GR. Physiology and healing dynamics of chronic cutaneous wounds. Am J Surgery. 1998; 176: 265.

2. Herroeder S, Durieux ME, Hillman MW. Inflammatory responses after surgery. Hosp Med. 2002; 63: 99-103.

3. Child DR, Murthy AS. Overview of wound healing and management. Surg Clin North Am. 2017; 97: 189-207.

4. Schaffer CJ, Nanney LB. Cell biology and wound healing. Int Rev Cytol. 1996; 169: 151-181.

5. Miron-Mendoza M, Graham E, Manohar S, Petroll WM. Fibroblast-fibronectin patterning and network formation in 3D fibrin matrices. Matrix Biol. 2017

6. Johnson MB, Pang B, Gardner DJ, Niknam-Benia S, Soundarajan V, Bramos A, et al. Topical fibronectin improves wound healing of irradiated skin. Sc Rep. 2017; 7: 3876.

7. Gueldner J, Zhang F, Zechmann B, Bruce ED. Evaluating a novel oxygenating therapeutic for its potential use in the advancement of wound healing. Toxicolln Vitro. 2017.

8. Pierce GF, Mustoe TA, Altrock BW, Deuel TF, Thomason A. Role of platelet -derived growth factor in wound healing. J Cell Biochem. 1991; 45: 319-326.

9. Cooper D, Yu EZ, Hennessey P, Ko F, Robson MC. Determination of endogenous cytokines in chronic wounds. Ann Surgery. 1994; 219: 688-692.

10. Koveker GB. Growth factors in clinical practice. International J of Clinical Practice. 2000; 5: 590-593.

11. Gaut D. Scars and Contractures. Surgery. 1999; 17: 73-75.

12. Weledji EP, Ngwane S. The management of keloids and hypertrophic scars. Darlington and County. Durham Medical Journal. 2012; 6: 39-45.

13. Flint $\mathrm{MH}$. The biological basis of Langer's lines. In Longacre JJ (ed); The ultra structure of collagen. Springfield, Charles C Thomas. 1976; 132-140.

14. McGregor EA. Fundamental techniques of Plastic Surgery. Churchill Livingstone, Edinburgh. 1995.

15. Yannas IV, Tzeranis DS, So PTC. Regeneration of injured skin and peripheral nerves requires control of wound contraction, not scar formation. Wound Repair Regen. 2017; 25: 177-191.

16. Claes L, Recknagel S, Ignatius A. fracture healing under healthy and inflammatory conditions. Nat Rev Rheumatol. 2012; 8: 133-143.

17. Benga A, Zor F, Korkmaz A, Marinescu B, Gorantla V. The neurochemistry of peripheral nerve regeneration. Indian J Plast Surg. 2017; 50: 5-15.

18. Diogo CC, Camassa JA, Pereira JE, Costa LMD, Filipe V, Couto PA, et al.
The use of sheep as a model for studying peripheral nerve regeneration following nerve injury: review of the literature. Neurol Res. 2017; 11: 1-14.

19. Irvin TT, Goligher JC. Aetiology of disruption of intestinal anastomosis. Br J Surg. 1973; 60: 461-464.

20. Weledji EP, VerlaV. Failure to rescue patients from early critical complications of oesophagogastric cancer surgery. Ann Med Surg (Lond). 2016; 7: 34-41.

21. Weledji EP, Ngowe NM. Surgical measures to reduce surgical site infection in open colorectal surgery. East Cent. Afr J Surg. 2013; 18: 1

22. Association of Coloproctology of Great Britain and Ireland. Guidelines for the management of colorectal cancer, 3rdedn. Association of Coloproctology of Gt Britain and Ireland. 2007.

23. Leslie A, Steele RJC. The interrupted serosubmucosal anastomosis. Still the gold standard. Colorectal Disease. 2003; 5: 362-366.

24. Leaper DJ, Harding K, Gleds. Wounds, Biology and management. Oxford Medical Edinburgh. 1998.

25. Jenkins TPN. The burst abdominal wound: a mechanical approach. British Journal of Surgery. 1976; 63: 873-876.

26. Mukherjee K, Kavalukas SL, Barbul A. Nutritional aspects of gastrointestinal wound healing. Adv Wound Care (New Rochelle). 2016; 5: 507-515.

27. Weledji EP, Kamga HLF, Assob JC, Nsagha DS. A critical review of HIVIAIDS and wound care. AFR J Cln Exper Microbiol. 2012; 13: 66-73.

28. Appleton GVN, Leaper DJ. The infected wound. Hospital Update. 1992; 1 189-198.

29. Li WW, Carter MJ, Mashiach E, Guthrie SD. Vascular assessment of wound healing: a clinical review. Int Wound J. 2017; 14: 460-469.

30. Thompson C, Fuhrmann MP. Nutrients and wound healing; still searching for the magic bullet. Nutrition in clinical practice. 2005; 20: 331-347.

31. Kogan S, Sood A, Garnick MS. Zinc and wound healing: A review of Zinc physiology and clinical applications. Wounds. 2017; 29: 102-106.

32. Wilte MB, Barbul A. General principles of wound healing. Surg Clin N Am. 1997; $77:$ 509-528.

33. Vermeulen H, Ubbink DT, Goossens A, de Vos R, Legemate DA. Systemic review of dressings and topical agents for surgical wound healing by secondary intention. B J Surg. 2005; 92: 665-672.

34. Moore LJ, Moore FA, Todd RS. Sepsis in General Surgery. 2010; 14: 611622.

35. Clarke JA. HIV transmission and Skin grafts. The Lancet. 1987; 329: 983.

36. Cooper ML, Spielvojeel R. Artificial Skin for wound healing. Clinics in Dermatology. 1994; 12: 183-191.
Austin J Surg - Volume 4 Issue 3 - 2017

ISSN : 2381-9030 | www.austinpublishinggroup.com

Weledii. (C) All rights are reserved
Citation: Weledji EP. Perspectives on Wound Healing. Austin J Surg. 2017; 4(3): 1104. 